EXTENDED REPORT

\title{
Characterisation of corneal fibrotic wound repair at the LASIK flap margin
}

\author{
A Ivarsen, T Laurberg, T Møller-Pedersen
}

Br J Ophthalmol 2003;87:1272-1278

See end of article for authors' affiliations

......................

Correspondence to: Torben Møller-Pedersen, Department of

Ophthalmology Aarhus

University Hospital

Nørrebrogade 44

DK-8000 Aarhus C,

Denmark;

tmp@akhphd.au.dk

Accepted for publication 7 April 2003
Aim: To characterise temporal changes in corneal wound repair at the LASIK flap margin.

Methods: 18 rabbits received monocular LASIK and were evaluated during 6 months using slit lamp and in vivo confocal microscopy. In three corneas, the exposed stroma was stained with DTAF. At various time points, corneas were processed for histology and stained for nuclei, f-actin, ED-A fibronectin, $\alpha$-smooth muscle actin, TGF- $\beta 1$, TGF- $\beta 2$, TGF- $\beta$ receptor II, and CTGF.

Results: At day 1, leucocytes migrated from the conjunctival vessels into the cornea. Near the limbus, the leucocytes were organised in long chains stretching towards the flap edge. From day 4, elongated fibroblasts migrated from the periphery to align in a circumferential band (approximately $250 \mu \mathrm{m}$ wide) next to the flap edge. The lateral extension of this stromal band was delimited by the incisional gap in the epithelial basement membrane. TGF- $\beta 1$, TGF- $\beta 2$, TGF- $\beta$ receptor II, and CTGF were expressed in the band from day 2. Myofibroblasts were identified at week 3 and over time a $50 \mu \mathrm{m}$ thick layer of fibrotic matrix was deposited. Concurrently, the peripheral circumferential band became narrower (width decreasing to $33 \%$ (SD 7\%) at 4 months; $n=5$ ) and showed an increased organisation with a gradual decline in reflectivity. At all time points, keratocytes within and below the flap remained quiescent and only minimal fibrosis developed at the interface.

Conclusions: Fibrotic wound repair following LASIK is restricted to a narrow band peripheral to the corneal flap edge. The lateral extension of the fibrosis is sharply delimited by the incisional gap in the epithelial basement membrane. The fibrotic wound healing at the LASIK flap margin is associated with myofibroblast transformation and wound contraction and involves a TGF- $\beta$ signalling pathway.
$\mathrm{L}$ aser in situ keratomileusis (LASIK) is a modern refractive surgical procedure that involves temporary displacement of a hinged corneal tissue flap during laser treatment of the underlying stromal bed. LASIK is associated with a high risk of complications related to the corneal flap including epithelial ingrowth, flap melting, and traumatic flap dislocation. $^{1-4}$ These complications may be related to the clinically observed lack of corneal wound healing that allows the corneal flap to be separated from its stromal bed for an indeterminate time after surgery. Thus, the LASIK interface is readily accessible for re-treatment more than 1 year after the initial procedure, ${ }^{5}$ and corneal flap dehiscence has been observed several years after uneventful surgery. ${ }^{4}$ Using slit lamp biomicroscopy, a white reflecting band is typically observed in the corneal flap periphery after LASIK (Fig 1). ${ }^{26}$ Over time, this circumferential band undergoes characteristic changes in width, texture, and reflectivity. The band is generally believed to represent corneal wound healing ${ }^{2-10}$; however, its exact nature, localisation, and significance have not been thoroughly investigated. Using a rabbit eye model, the present study characterises temporal changes in corneal wound repair at the LASIK flap margin. Various components of the wound healing response are studied, including initial inflammation, TGF- $\beta$ growth factor expression, keratocyte phenotypic transformations, deposition of extracellular matrix (fibrosis), and wound contraction.

\section{MATERIALS AND METHODS}

\section{Study design}

Eighteen New Zealand White rabbits (weight 4.0-5.5 kg) with normal corneas received monocular LASIK and were evaluated during 6 months using slit lamp and in vivo confocal microscopy. In three corneas, the exposed stroma was vitally stained during surgery with 5-(4,6-dichlorotriazinyl) aminofluorescein (DTAF; Sigma, Denmark); a green fluorescent probe that binds covalently to collagen, allowing detection of unstained, newly deposited matrix. ${ }^{11}$ Before surgery and at all examinations, animals were anaesthetised topically with oxybuprocaine $0.8 \%$, and systemically with midazolam $2.0 \mathrm{mg} / \mathrm{kg}$ and Hypnorm $0.3 \mathrm{ml} / \mathrm{kg}$ (fentanyl $0.315 \mathrm{mg} / \mathrm{ml}$, and fluanisone $10 \mathrm{mg} / \mathrm{ml}$; Janssen Pharmaceuticals, Beerse, Belgium). Preoperatively, two drops of ciprofloxacin $3 \mathrm{mg} / \mathrm{ml}$ and chloramphenicol $0.5 \%$ were applied. Postoperative treatment (three times per day) included topical ciprofloxacin for 2 days and topical chloramphenicol and systemic buprenorphine $(0.05 \mathrm{mg} / \mathrm{kg})$ for 5 days. At selected time points, animals were killed by injecting sodium pentobarbital $150 \mathrm{mg} / \mathrm{kg}$, and the corneas were processed for histology. The study was approved by the Danish Animal Experiments Inspectorate, and all animals were housed and treated according to the ARVO statement for the use of animals in ophthalmic and visual research.

\section{Surgery}

One week before LASIK, the nictating membrane was removed in all eyes. During LASIK surgery, the eye was gently proptosed and a hinged corneal flap was cut using a microkeratome (Supratome; Schwind, Kleinostheim, Germany). The flap was lifted and the stromal bed received a $6 \mathrm{~mm}$ diameter, -8.0 dioptres correction using a MEL 70 G-Scan excimer laser (Asclepion, Jena, Germany). After careful repositioning of the flap, a bandage soft contact lens ( $7.4 \mathrm{~mm}$ radius of curvature; $\mathrm{Dk} / \mathrm{t}=27$; Igel $\mathrm{Rx} 67$ Spheric UV; Ultravision International, Beds, UK) was inserted for 2 days to prevent flap dislocation. In three corneas, DTAF staining was performed at surgery by irrigating the flap and stromal bed with a sterilised solution ( $\mathrm{pH} 7.4$ ) of $0.5 \%$ DTAF 

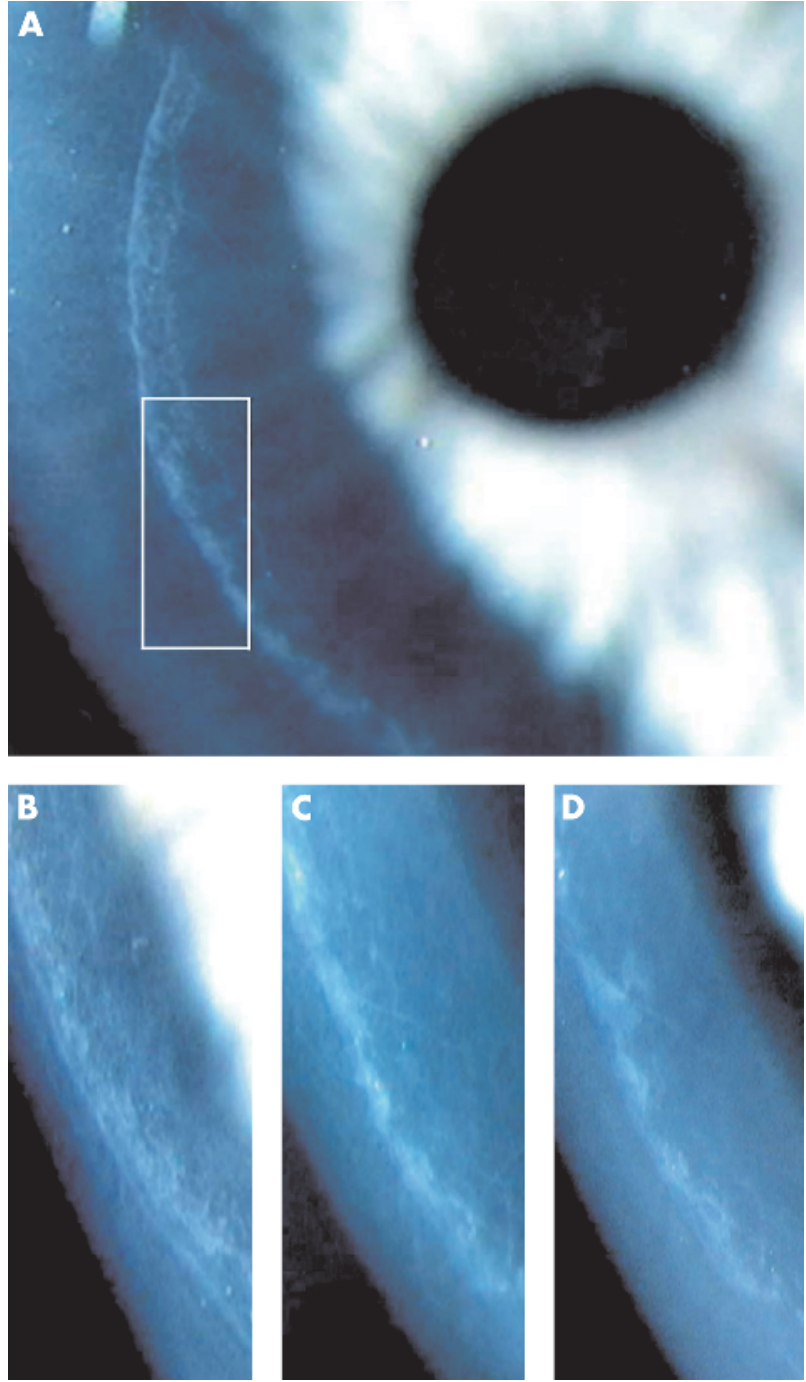

Figure 1 Slit lamp biomicroscopy of the flap margin in a LASIK patient demonstrating a white reflecting circumferential band 6 months postsurgery (A). To illustrate temporal changes in morphology, the band is shown at higher magnification (rectangle) at 1 month (B), 6 months $(C)$, and 12 months (D). Note the characteristic changes in width, texture, and reflectivity.

dissolved in $0.2 \mathrm{M}$ sodium bicarbonate. After 1 minute, the stained surfaces were rinsed with sterile saline and the flap was repositioned.

\section{Slit lamp and in vivo confocal microscopy}

All rabbits were evaluated preoperatively using slit lamp and in vivo confocal microscopy as previously reported..$^{12}$ After surgery, the flap margin and adjacent regions were examined daily for the first week, then at 1, 2, 3, and 4 weeks, and at 2, 4 , and 6 months. At each time point, a minimum of two rabbits was evaluated. However, to avoid alteration of the wound healing response, the same animal was not examined on two consecutive days during the first week. In a group of five animals, the same region of the flap margin was photographed at week 1, 2, 8, and 16 using slit lamp biomicroscopy. Subsequently, the relative width of the peripheral circumferential band was measured using digital image analysis (two measurements at each time point). Using in vivo confocal microscopy, three dimensional surface projections of through-focusing $\mathrm{z}$-series of the flap edge were generated, and representative two dimensional and three dimensional images were contrast adjusted.

\section{Histology}

Corneal tissue was obtained for histology at day 2 and 4, and at week 1, 2, and 3 post-LASIK ( 10 animals in total with two rabbits at each time point). Five corneas were processed for histology at 6 months as were the three DTAF stained corneas. The tissue was fixed in situ by anterior chamber perfusion (4\% formaldehyde for 3 minutes), excised, dissected, embedded in Tissue-Tek (Sakura, Tokyo, Japan), snap frozen in liquid nitrogen, and stored at $-80^{\circ} \mathrm{C}$. Serial cryostat cross sections (thickness approximately $5 \mu \mathrm{m}$ ) were cut, air dried, and fixed in acetone for 10 minutes. To detect $\mathrm{f}$-actin, sections were stained with Alexa Fluor 568 conjugated phalloidin $(1.1 \mu \mathrm{mol} / \mathrm{l}$ for 3 hours; Molecular Probes, Leiden, Netherlands). Fibronectin and $\alpha$-smooth muscle actin ( $\alpha$-SMA) were detected by incubating sections overnight with mouse anti-human ED-A fibronectin (clone DHl; 1:200; Biotrend, Cologne, Germany), respectively mouse antihuman $\alpha$-SMA (clone 1A4; 1:400; Sigma, Denmark). To detect selected growth factors and receptors, sections were incubated overnight with one of the following four primary antibodies: goat anti-human transforming growth factor $\beta 1$ (TGF- $\beta 1$; $1: 100$; Santa Cruz Biotechnology, CA, USA); mouse anti-human transforming growth factor $\beta 2$ (TGF- $\beta 2$; clone 8607.211; 1:75; R\&D Systems, Minneapolis, MN, USA); goat anti-human transforming growth factor $\beta$ receptor II (TGF$\beta$ RII; 1:100; Santa Cruz Biotechnology, CA, USA); and goat anti-human connective tissue growth factor (CTGF; 1:12500; a generous gift from Dr Gary Grotendorst). ${ }^{13}$ Primary antibodies were visualised with one of the following Alexa Fluor 568 conjugated antibodies (Molecular Probes, Leiden, Netherlands): goat anti-mouse IgG (1:100 for 30 minutes) and donkey anti-goat IgG (1:100 for 30 minutes). Colocalisation of cell nuclei was performed using Hoechst 33342 ( $2 \mu \mathrm{g} / \mathrm{ml}$; Molecular Probes, Leiden, Netherlands). Control experiments included evaluation of tissue from unoperated animals, use of unspecific primary antibodies, omission of primary or secondary antibodies, and preadsorption of primary antibodies with corresponding growth factors (to ensure specificity). Sections were evaluated using a Zeiss Axiovert 135 inverted microscope, equipped with a $20 \times$ objective $(\mathrm{NA}=0.75)$ and a zoom adaptor (range $0.4-2.0 \times)$. Selected images were overlaid and contrast adjusted.

\section{RESULTS}

\section{Slit lamp biomicroscopy}

Throughout the study, no dislocation of the LASIK flaps was observed. However, immediately after surgery a narrow circumferential gap was identified along the flap edge (Fig 2A). Over time, characteristic changes in the morphology and reflectivity of this region were detected. During the first week, a well defined circular band (approximately $1 / 4 \mathrm{~mm}$ wide) appeared that in the following weeks became increasingly reflective and developed a fibrillar texture (Fig 2B). By 2 months, a gradual condensation had occurred and the band seemed more organised (Fig 2C). At 4 and 6 months, the flap edge reflectivity had decreased considerably, leaving only a low reflective region (Fig 2D). Over time, the circular band gradually became narrower (Fig 2E), measuring 100\% at l week, $89 \%$ (SD 10\%) (2 weeks), 53\% (13\%) (8 weeks), and $33 \%(7 \%)$ ( 16 weeks $)(n=5$; sample means different at all time points; analysis of variance; $\mathrm{p}<0.05)$. The temporal changes in width, texture, and reflectivity at the LASIK flap edge appeared to parallel those observed in humans (compare Fig 2 with Fig 1), suggesting that the rabbit may provide an acceptable model for LASIK surgery. 

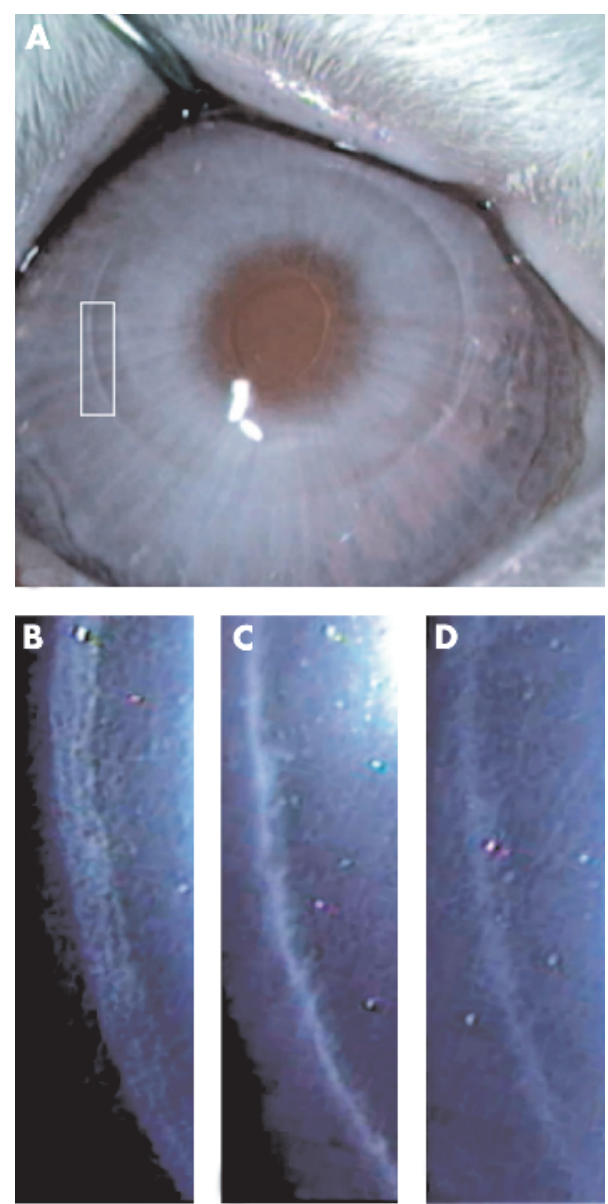

E

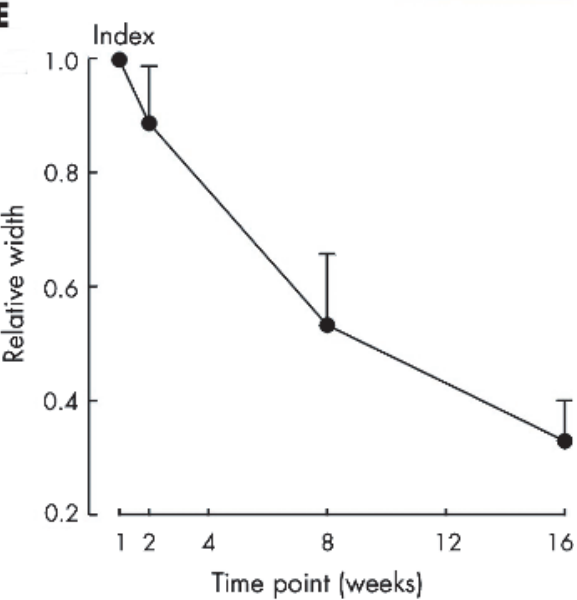

Figure 2 Slit lamp biomicroscopy of a rabbit cornea 2 weeks postLASIK (A). Part of the flap margin (rectangle) is shown at higher magnification. At 2 weeks (B), a white reflecting circumferential band with a fibrillar texture (approximately $1 / 4 \mathrm{~mm}$ wide) is seen. By 2 months (C), this band appears more organised with a marked increase in reflectivity, and at 6 months (D), the band is only weakly reflecting. Quantitative measurement of the width of the circumferential band (E) demonstrates a gradual decrease from $100 \%$ at 1 week to $33 \%(7 \%)$ at 16 weeks (mean $(S D) ; n=5$ ). The small bright objects in (B-D) represent particles in the tear film.

\section{In vivo confocal microscopy}

\section{Conjunctival and corneal Inflammation}

In the conjunctival vessels, numerous leucocytes were observed 24 hours after LASIK. Careful examination revealed that these cells appeared to slow down, adhere to the vessel wall, and migrate into the surrounding tissue (Fig 3A, arrowheads). Near limbus, multiple inflammatory cells were found in the anterior $40 \mu \mathrm{m}$ stroma (Fig 3B). A noteworthy observation was the presence of long chains of inflammatory cells stretching from the periphery towards the microkeratome entry (Fig 3C); suggesting directional migration of leucocytes. The leucocytes were exclusively located peripherally to the flap edge and were not observed centrally, within, or below the flap. The inflammatory response had almost disappeared by day 2 .

\section{Flap edge morphology}

From day four, spindle-shaped cells (Fig 4A, arrows) in the anterior stroma began to align in a circumferential band next to the flap edge. These elongated cells first appeared in the periphery, suggesting cellular transformation and migration of the adjacent peripheral keratocytes. By contrast, more centrally located cells within and below the flap remained quiescent (curved arrows). At 2 weeks post-LASIK, the peripheral circumferential band (measuring approximately $250 \mu \mathrm{m}$ in width and $25 \mu \mathrm{m}$ in depth) showed further organisation and a marked increase in reflectivity, corresponding to the biomicroscopic findings (compare Fig 4B with Fig $2 \mathrm{~B}$ ). This increase in light scattering appeared to be caused by closely packed spindle-shaped cells (Fig 4B, arrows) and deposition of extracellular material. In contrast, the adjacent cells (curved arrows) on both sides of the peripheral circumferential band appeared quiescent. Over time, the band became narrower and more organised, and the reflectivity gradually declined. Thus, at 6 months, quiescent keratocytes (Fig 4C, curved arrows) were observed in a moderately reflective extracellular matrix.

\section{Basement membrane}

At day 1 post-LASIK, the epithelial defect at the incision had healed. However, below the intact epithelium, an outer (Fig 5A, arrows) and an inner break (Fig 5B, arrows) in the basement membrane was identified; corresponding to the microkeratome entry. These sharply defined interruptions in the basement membrane were separated by a gap that delimited the lateral extension of the underlying stromal wound repair (Fig 5C, D). This noteworthy observation was further supported by a 3D reconstruction of the flap edge region (Fig 6) that clearly demonstrates the spatial relation between the basement membrane and the wound repair within the peripheral circumferential band.

\section{Histology}

At the flap margin, no major acellular zones were detected in the stroma at any time point. From week l post-LASIK, elongated cells with a prominent f-actin expression (Fig 7A, curved arrows) were noted between the incisional breaks in the basement membrane (arrowheads). These migratory cells stretched from the underlying and peripheral stroma, similar to the in vivo observations (compare Fig 7A to Fig 4A). Corresponding to the f-actin staining, expression of ED-A fibronectin was detected. By 3 weeks, f-actin (Fig 7B) and fibronectin (Fig 7C) were expressed in the subepithelial region, and elongated cells were no longer present in the underlying stroma, suggesting that cell migration from the periphery had ended. Concurrently, expression of $\alpha$-SMA was noted, consistent with myofibroblast transformation. Serial sectioning indicated that the $\alpha$-SMA expression (Fig 7D) was restricted to a minor part of the f-actin and fibronectin positive regions (compare Fig 7D with Fig 7B, C). At 6 months, expression of ED-A fibronectin or $\alpha$-SMA was no longer detected, and f-actin showed only weak staining similar to the normal unwounded cornea. Still, the wound repair zone was easily identified by a prominent epithelial 

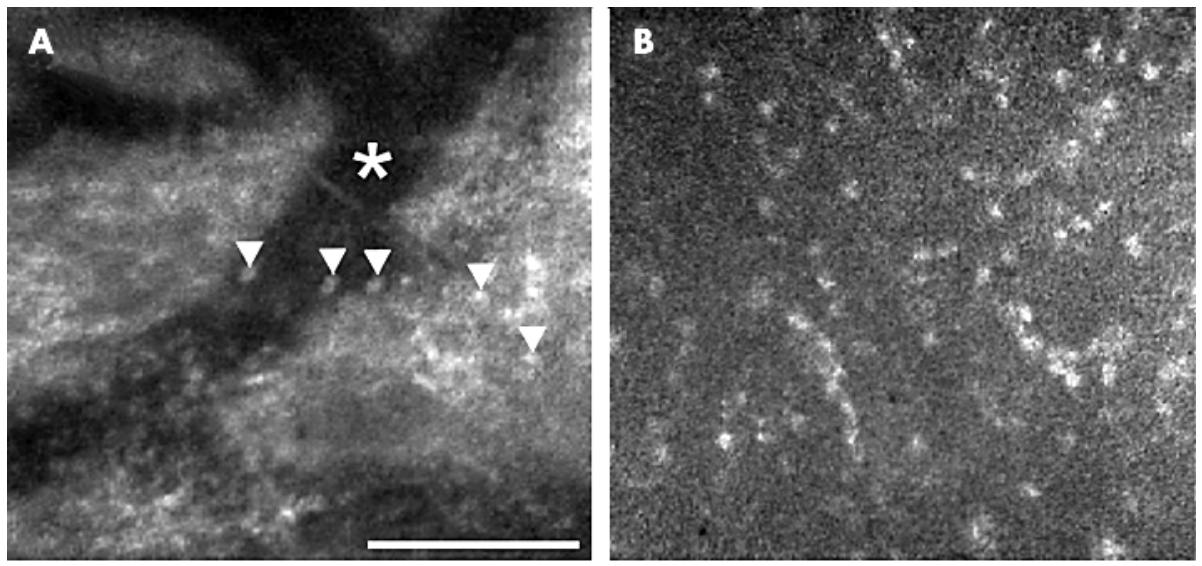

Figure 3 In vivo confocal microscopy of the conjunctiva and corneal periphery 24 hours after LASIK. (A) Numerous inflammatory cells (arrowheads) roll and adhere to the conjunctival vessels (asterisk) followed by extravasation into the surrounding tissue. (B) Accumulation of leucocytes in the anterior corneal stroma near limbus. (C) Montage of confocal images from the corneal stroma showing a long chain of inflammatory cells stretching from the corneal periphery towards the flap edge (arrows). The abrupt changes in image intensity are due to contrast variations in neighbouring images. Bar indicates $100 \mu \mathrm{m}$.

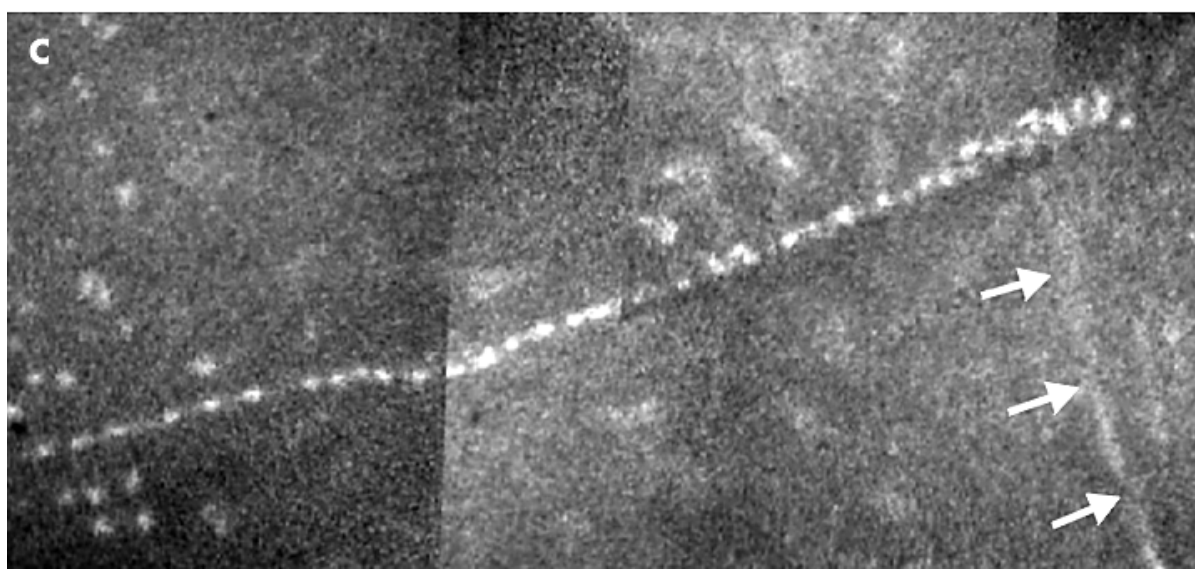

hyperplasia (up to 10 layers compared to the approximately five layers in the unwounded cornea; Fig 7). Despite this hyperplasia, epithelial ingrowth was not seen at any time point. At 6 months, an unstained, subepithelial region (approximately $50 \mu \mathrm{m}$ thick) was detected in DTAF labelled corneas (Fig 7E), demonstrating stromal fibrosis peripheral to the flap edge. By contrast, very little unstained tissue was found at the LASIK interface (arrowhead). Correspondingly, the central cornea showed a normal expression of f-actin and no staining for $\alpha$-SMA at any time point.

No expression of TGF- $\beta 1$, TGF- $\beta 2$, TGF- $\beta$ RII, or CTGF was detected in the unwounded stroma of the normal rabbit cornea. Two days after LASIK, TGF- $\beta 1$ (Fig 8A) and TGF- $\beta 2$ (Fig $8 \mathrm{~B}$ ) were expressed anteriorly in the stroma between the basement membrane breaks. Concurrently, TGF-BRII and CTGF were detected in the same region. At 2 and 3 weeks, the stromal expression of the three growth factors and TGF$\beta$ RII had narrowed and was restricted to a thin subepithelial layer within the wound repair zone (Fig 8C-E). Six months post-LASIK, the expression of all growth factors and TGF$\beta$ RII was negative, similar to that of the unoperated cornea. Throughout the study, no expression of TGF- $\beta 1$, TGF- $\beta 2$, TGF- $\beta$ RII, or CTGF was detected at the LASIK interface or in the stroma within or below the flap. In the epithelium and endothelium of the preoperative cornea TGF- $\beta 1$, TGF- $\beta 2$, TGF- $\beta$ RII, and CTGF were weakly expressed. After LASIK, no major changes in the epithelial and endothelial expression of the three growth factors and the receptor could be identified.

\section{DISCUSSION}

The present study demonstrates that post-LASIK fibrotic wound repair in the rabbit cornea is restricted to a
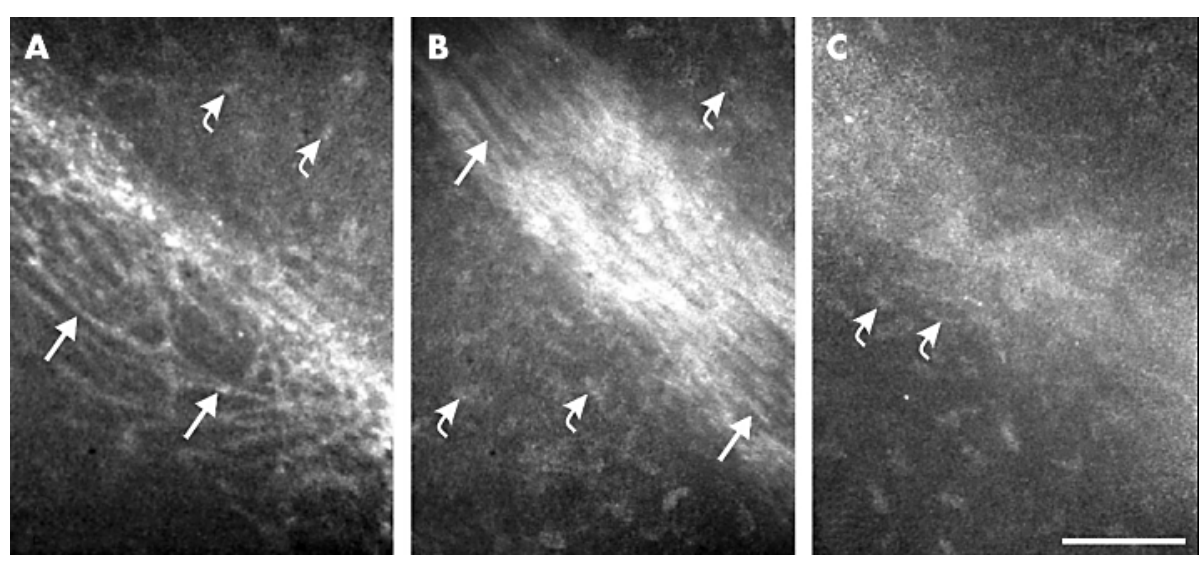

Figure 4 In vivo confocal microscopy of the LASIK flap edge within the rectangle shown in Figure.2A. (A) At day 4 , spindle-shaped fibroblasts (arrows) stretch from the periphery towards the flap edge, while keratocytes (curved arrows) within the flap remain quiescent. (B) Two weeks post-LASIK, the spindle-shaped fibroblasts (arrows) are organised circumferentially in a highly reflective matrix. Normal appearing keratocytes (curved arrows) are present on both sides of the wound repair zone. (C) By 6 months, quiescent keratocytes (curved arrows) are observed in a moderately reflective matrix. Bar indicates $100 \mu \mathrm{m}$. 

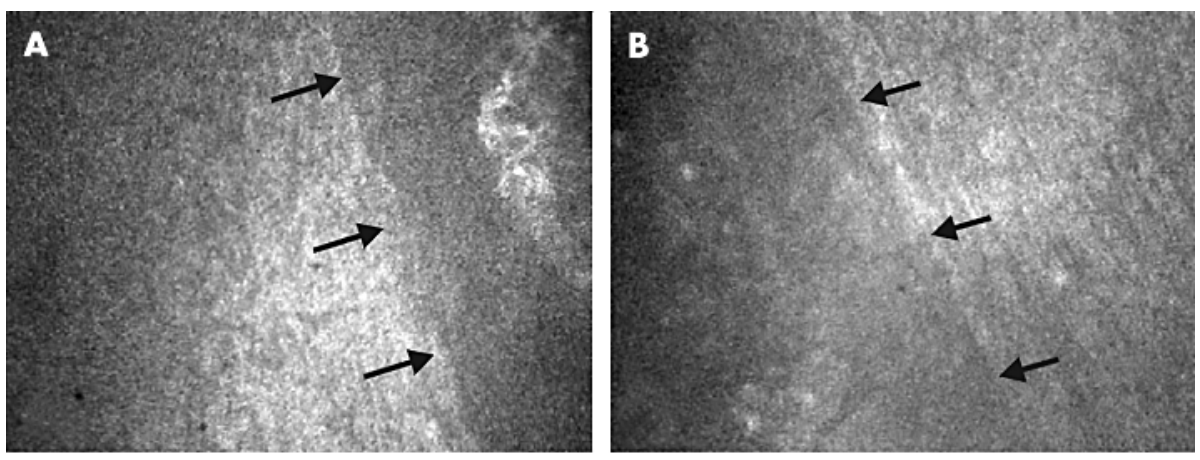

Figure 5 In vivo confocal microscopy at the flap margin 5 days post-LASIK. An outer (A) and an inner (B) break (arrows) in the basement membrane delimit the microkeratome entry. In the underlying stroma $(C, D)$, the lateral extension of the reflective wound repair is restricted to the region between the breaks in the basement membrane (arrows). Bar indicates $100 \mu \mathrm{m}$.
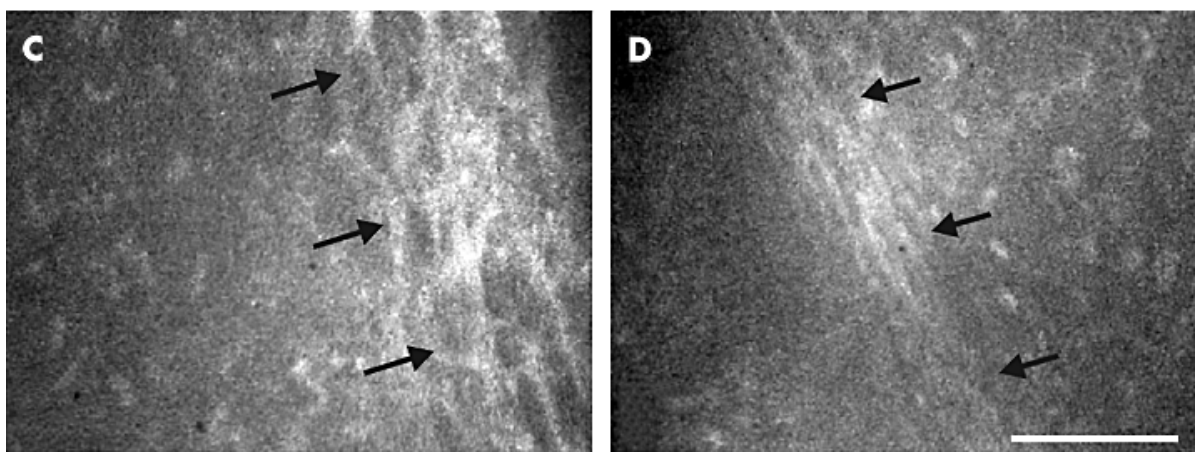

circumferential band in the anterior stroma between the incisional breaks in the epithelial basement membrane. The development of fibrosis at the flap edge is preceded by a characteristic sequence of events, beginning with an initial influx of inflammatory cells. Thus, at 24 hours post-LASIK rolling, adhesion, and extravasation of leucocytes were observed in the conjunctival vessels; corresponding to the recent observations in humans. ${ }^{14}$ Near limbus, these inflammatory cells were organised in long chains, indicating directional migration towards the microkeratome incision. A similar organisation of leucocytes following corneal wounding has previously been recognised by Wolter and hypothesised to represent migration in preformed spaces. ${ }^{15}$ The directional migration and accumulation of leucocytes next to the LASIK flap edge suggest that proinflammatory cytokines and chemokines are present in this region. Evidence of such signalling molecules has been found in the tear fluid, ${ }^{16}$ epithelium, ${ }^{17} 18$ and in keratocytes. ${ }^{18}{ }^{19}$ By contrast, the lack of leucocytes at the LASIK interface may indicate that an isolated stromal injury induces less of a chemotactic signal than when the epithelium and its basement membrane are involved. Accordingly, previous studies have showed lack of inflammation following manual epithelial debridement compared to an intense inflammation after basement membrane disruption (caused by a transepithelial photoablation including a $14 \mu \mathrm{m}$ stromal keratectomy). ${ }^{20}$ In the intact cornea, the epithelial basement membrane has been reported to bind cytokines, ${ }^{21}{ }^{22}$ suggesting that it may act as a barrier for signaling molecules from the epithelium or tear fluid. ${ }^{23}$ Thus, when the basement membrane integrity is compromised at the LASIK flap edge, signalling molecules with mitogenic and chemotactic effect on keratocytes ${ }^{22} 24$ and inflammatory cells ${ }^{16-18}$ may enter the stroma. The present finding of directional cell migration and localised activation of keratocytes (between the breaks in the basement membrane) supports this hypothesis.

In the normal cornea, TGF- $\beta 1$, TGF- $\beta 2$, TGF- $\beta$ receptor II, and CTGF were expressed in the surface epithelium, whereas no signal was detected in the unwounded stroma. Following LASIK, activation of a TGF- $\beta$ signalling pathway was

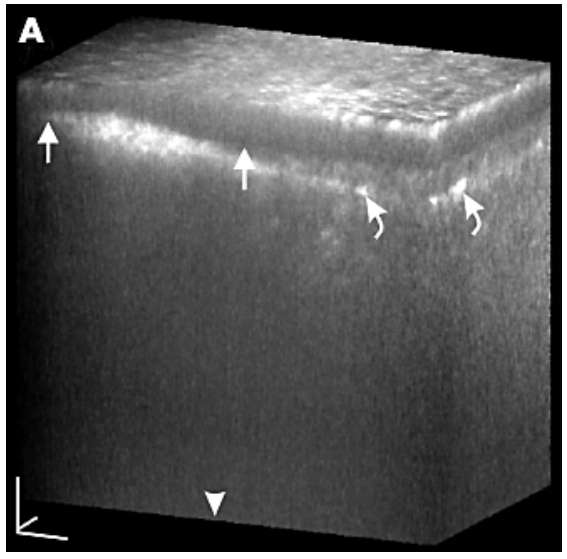

B

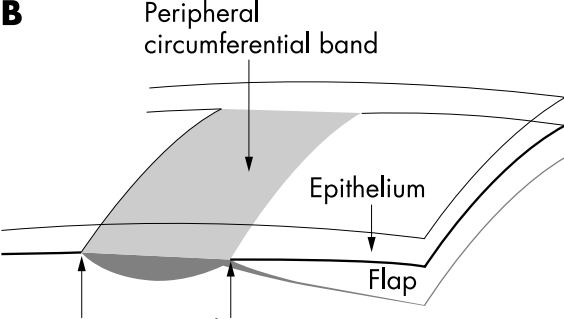

Basement membrane
Figure 6 Three dimensional reconstruction (A) and diagram (B) of the flap edge region 2 weeks postLASIK. An outer (left arrow) and an inner (right arrow) break in the basement membrane sharply delimit the stromal wound repair. In contrast, the interface (below the LASIK flap) is weakly reflecting except for a few bright interface particles (curved arrows). It should be noted that the high reflectivity of the anterior stroma causes two minor artefacts when imaged by confocal microscopy: (1) the $z$-axis extension of the wound repair is exaggerated leading to an apparent bulging up into the epithelium; (2) the cellular detail within the deeper cornea is faint due to loss of signal. The arrowhead indicates the position of the endothelium, and the three dimensional bar represents $50 \mu \mathrm{m}$. 

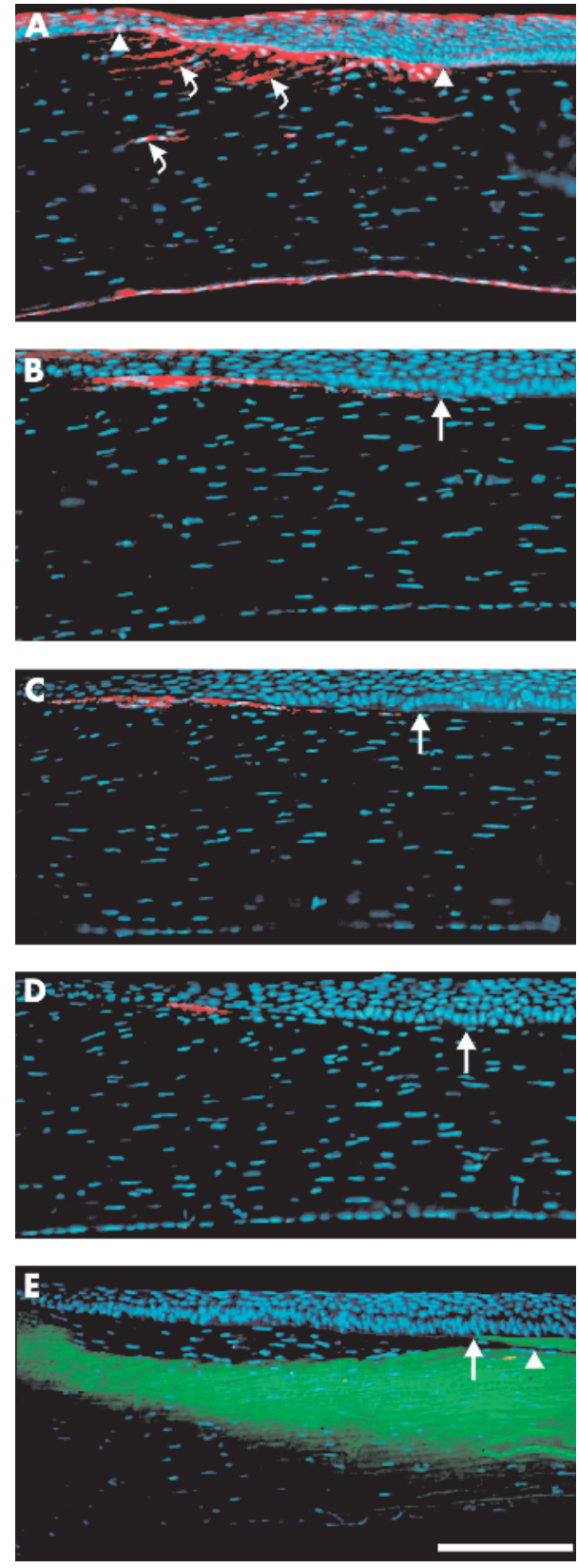

Figure 7 Fluorescence microscopy of the LASIK flap edge at 1 week (A), 3 weeks (B-D), and 6 months (E) post-surgery. All images are oriented with the corneal periphery to the left. (A) Co-localisation of f-actin (red; phalloidin) and nuclei (blue; Hoechst) demonstrating elongated cells with a prominent f-actin expression (curved arrows). These cells stretch from the underlying and peripheral stroma to align in a wound repair zone, located between the incisional breaks in the basement membrane (arrowheads). (B-D) Serial cross sections stained for f-actin ( $B$; red), ED-A fibronectin (C; red), $\alpha$-SMA (D; red), and counterstained for nuclei (blue). Note the coinciding expression of f-actin and ED-A fibronectin in the wound repair zone as well as the expression of $\alpha$-SMA in a minor part of this zone. (E) DTAF stained (green) cornea (counterstained for nuclei; blue) demonstrating a large unstained region peripheral to the flap edge (arrow). By contrast, only minimal deposition of new tissue is observed at the LASIK interface (arrowhead). At all time points, the epithelium covering the wound repair zone and the flap edge is hyperplastic (as seen on the right side of the images), contrary to the normal epithelium in the adjacent regions (as demonstrated on the left side of the images). Straight arrows indicate the position of the flap edge. Bar indicates $100 \mu \mathrm{m}$.

detected in the corneal stroma next to the flap edge. The expression included TGF- $\beta$ receptor II (which is mandatory
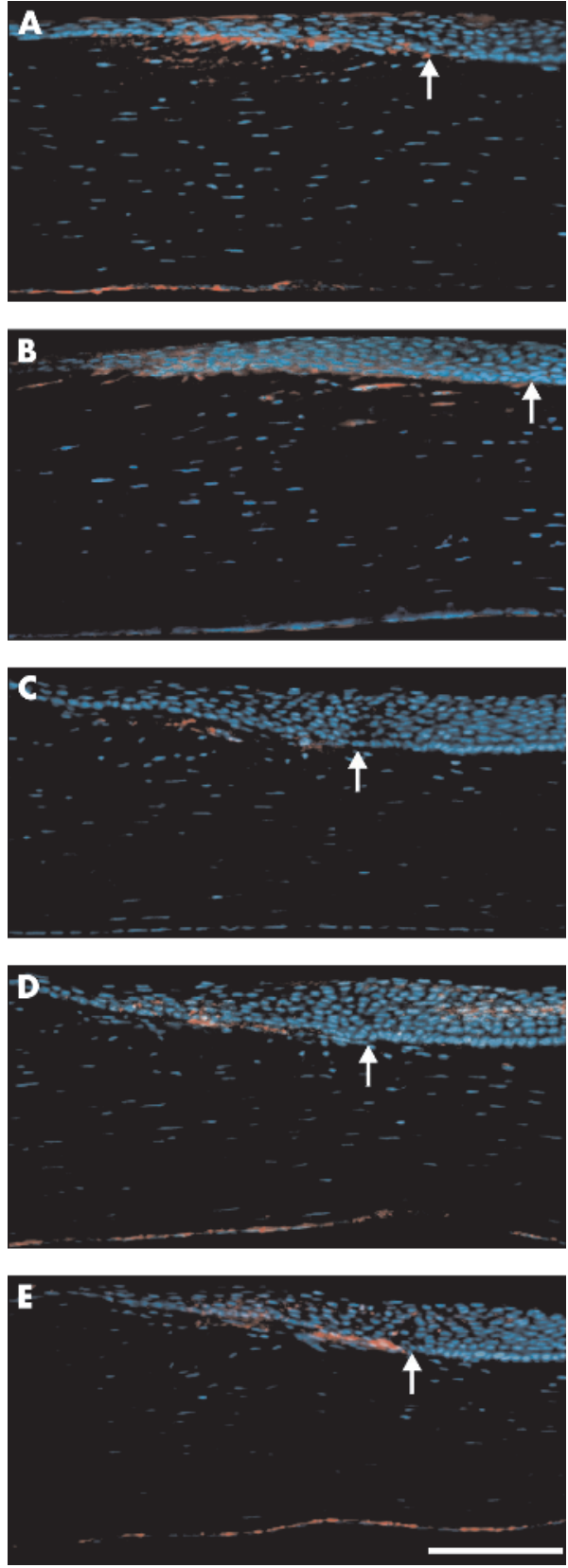

Figure 8 Immunohistochemistry of the LASIK flap edge (arrows) demonstrating the expression of TGF- $\beta 1$ (A; red) and TGF- $\beta 2$ (B; red) at 4 days, and TGF- $\beta 2$ (C; red), TGF- $\beta R I I$ ( $D$; red), and CTGF (E; red) at 2 weeks post-surgery. All sections are counterstained for nuclei (blue), and oriented with the corneal periphery to the left. (C-E) Represent serial cross sections. Bar indicates $100 \mu \mathrm{m}$.

for TGF- $\beta$ signal transduction) in the keratocytes, and TGF$\beta 1$, TGF- $\beta 2$, and CTGF between the basement membrane breaks from day 2 . Overall, these findings suggest that TGF- $\beta$ receptor II is upregulated in the keratocytes concurrent, with the release of TGF- $\beta$ into the corneal stroma. The localised expression of TGF- $\beta$ signalling molecules at the LASIK flap margin persisted for at least 3 weeks and was not detected by 6 months. The activation of TGF- $\beta$ may account for the observed myofibroblast transformation at week 3, in accordance with previous studies. ${ }^{112627}$ Moreover, the observed expression of ED-A fibronectin has been reported as essential for $\alpha$-SMA expression. ${ }^{28}$ Myofibroblasts are known to cause wound contraction following, for example, full thickness incisional wounds and radial keratotomy, leading to changes 
in corneal curvature. ${ }^{29-31}$ Thus, the present finding of myofibroblasts at the LASIK flap edge may indicate an active wound contraction in the region. The slit lamp detectable changes in the width of the peripheral circumferential band (Fig 2E) support this interpretation. Speculatively, such wound contraction may lead to tightening and flattening of the LASIK flap contributing to changes in the postoperative corneal refraction (possibly a relative hyperopic shift).

Besides the ability to induce myofibroblast transformation, TGF- $\beta 1$ and TGF- $\beta 2$ are generally accepted as fibrogenetic growth factors. ${ }^{27}{ }^{32}$ Also the importance of CTGF (a presumed downstream mediator of TGF- $\beta)^{32}{ }^{33}$ for the development of fibrosis has previously been recognised. ${ }^{34}{ }^{35}$ In the present study, the stromal expression of TGF- $\beta 1$, TGF- $\beta 2$, and CTGF indicate that all of these growth factors are involved in the fibrotic wound repair. The strict localisation of fibrosis peripheral to the LASIK flap edge, contrary to the minimal fibrosis below the entire flap, suggests that the LASIK interface in the rabbit cornea never heals. These findings in rabbits provide a structural explanation for the clinically observed lack of post-LASIK wound healing that allows the corneal flap to be separated from its stromal bed for an indeterminate time after surgery. ${ }^{45}$ Since the rabbit cornea otherwise is known to heal aggressively (following, for example, photorefractive keratectomy or a penetrating wound) ${ }^{36-38}$ LASIK provides an interesting model for studying the basic regulation of corneal wound repair and the development of fibrosis. Further insight into these important aspects is required to improve the outcome of LASIK and other corneal refractive surgical procedures.

\section{ACKNOWLEDGEMENTS}

The authors thank Gregory S Schultz and Gary Grotendorst for providing the CTGF antibody, and Poul Rostgaard for assistance with immunohistochemistry.

This work was supported in part by generous donations from The Faculty of Health Sciences at the University of Aarhus, The Danish Medical Research Council, The Danish Association for Prevention of Eye Diseases and Blindness, The Novo Nordisk Foundation, The Institute of Experimental Clinical Research at Aarhus University Hospital, The Synoptik Foundation, Ingeniør August Frederik Erichsens Legat, Ingrid Munkholms Legat, The Hørslev Foundation, Fonden til Lægevidenskabens Fremme, Alice og Jørgen A Rasmussens Memorial Grant, Jørgen Bagenkop Nielsens Myopia Foundation, The Research Foundation at the University of Aarhus, The Danish Eye Research Foundation, The Danish Medical Association Research Foundation, Svend H.A. Schrøders Foundation, The Toyota Foundation, Jacob Madsens Foundation, Ib Henriksens Foundation, The Anniversary Foundation for King Christian IX and Queen Louise.

\section{Authors' affiliations}

A lvarsen, T Laurberg, T Møller-Pedersen, Department of

Ophthalmology, Aarhus University Hospital, Denmark

\section{REFERENCES}

1 Wang MY, Maloney RK. Epithelial ingrowth after laser in situ keratomileusis. Am J Ophthalmol 2000;129:746-51.

2 Vesaluoma MH, Petroll WM, Perez-Santonja JJ, et al. Laser in situ keratomileusis flap margin: wound healing and complications imaged by in vivo confocal microscopy. Am J Ophthalmol 2000;130:564-73.

3 Perez-Santonja JJ, Bellot J, Claramonte P, et al. Laser in situ keratomileusis to correct high myopia. J Cataract Refract Surg 1997;23:372-85.

4 Iskander NG, Peters NT, Anderson PE, et al. Late traumatic flap dislocation after laser in situ keratomileusis. J Cataract Refract Surg 2001;27:1111-14.

5 Rashad KM. Laser in situ keratomileusis retreatment for residual myopia and astigmatism. J Refract Surg 2000;16:170-6.

6 El Danasoury MA, El Maghraby A, Klyce SD, et al. Comparison of photorefractive keratectomy with excimer laser in situ keratomileusis in correcting low myopia (from -2.00 to -5.50 diopters). A randomized study. Ophthalmology 1999;106:41 1-20.

7 Chang SW, Benson A, Azar DT. Corneal light scattering with stromal reformation after laser in situ keratomileusis and photorefractive keratectomy. J Cataract Refract Surg 1998;24:1064-9.
8 Amm M, Wetzel W, Winter M, et al. Histopathological comparison of photorefractive keratectomy and laser in situ keratomileusis in rabbits. J Refract Surg 1996; 12:758-66.

9 Perez-Santonja JJ, Linna TU, Tervo KM, et al. Corneal wound healing after laser in situ keratomileusis in rabbits. J Refract Surg 1998;14:602-9.

10 Wachtlin J, Langenbeck K, Schrunder S, et al. Immunohistology of corneal wound healing after photorefractive keratectomy and laser in situ keratomileusis. J Refract Surg 1999;15:451-8.

11 Moller-Pedersen T, Cavanagh HD, Petroll WM, et al. Neutralizing antibody to TGFbeta modulates stromal fibrosis but not regression of photoablative effect following PRK. Curr Eye Res 1998;17:736-47.

12 Ivarsen A, Stultiens BA, Møller-Pedersen T. Validation of Confocal Microscopy Through Focusing for Corneal Sublayer Pachymetry. Cornea 2002;21:700-4.

13 Duncan MR, Frazier KS, Abramson S, et al. Connective tissue growth factor mediates transforming growth factor beta-induced collagen synthesis: downregulation by CAMP. FASEB J 1999;13:1774-86.

14 Kirveskari J, Vesaluoma MH, Moilanen JA, et al. A novel non-invasive, in vivo technique for the quantification of leukocyte rolling and extravasation at sites of inflammation in human patients. Nat Med 2001;7:376-9.

15 Wolter JR. Reactions of the cellular elements of the corneal stroma. Arch Ophthalmol 1958;59:873-81.

16 Vesaluoma MH, Tervo T. Tenascin and cytokines in tear fluid after photorefractive keratectomy. J Refract Surg 1998;14:447-54.

17 Wilson SE, Schultz GS, Chegini N, et al. Epidermal growth factor, transforming growth factor alpha, transforming growth factor beta, acidic fibroblast growth factor, basic fibroblast growth factor, and interleukin-1 proteins in the cornea. Exp Eye Res 1994:59:63-71.

18 Li DQ, Tseng SC. Differential regulation of cytokine and receptor transcript expression in human corneal and limbal fibroblasts by epidermal growth factor, transforming growth factor-alpha, platelet-derived growth factor B, and interleukin-1 beta. Invest Ophthalmol Vis Sci 1996;37:2068-80.

19 Hong JW, Liu JJ, Lee JS, et al. Proinflammatory chemokine induction in keratocytes and inflammatory cell infiltration into the cornea. Invest Ophthalmol Vis Sci $2001 ; 42: 2795-803$.

20 Møller-Pedersen T, Cavanagh HD, Petroll WM, et al. Corneal haze development after PRK is regulated by volume of stromal tissue removal. Cornea 1998;17:627-39.

21 Soubrane G, Jerdan J, Karpouzas I, et al. Binding of basic fibroblast growth factor to normal and neovascularized rabbit cornea. Invest Ophthalmol Vis Sci 1990;31:323-33

22 Kim WJ, Mohan RR, Mohan RR, et al. Effect of PDGF, IL-l alpha, and BMP2/4 on corneal fibroblast chemotaxis: expression of the platelet-derived growth factor system in the cornea. Invest Ophthalmol Vis Sci 1999;40:1364-72.

23 Zieske JD, Mason VS, Wasson ME, et al. Basement membrane assembly and differentiation of cultured corneal cells: importance of culture environment and endothelial cell interaction. Exp Cell Res 1994:214:621-33.

24 Pancholi S, Tullo A, Khaliq A, et al. The effects of growth factors and conditioned media on the proliferation of human corneal epithelial cells and keratocytes. Graefes Arch Clin Exp Ophthalmol 1998;236:1-8.

25 Andresen JL, Ehlers N. Chemotaxis of human keratocytes is increased by platelet-derived growth factor-BB, epidermal growth factor, transforming growth factor-alpha, acidic fibroblast growth factor, insulin-like growth factorI, and transforming growth factor-beta. Curr Eye Res 1998;17:79-87.

26 Jester JV, Barry-Lane PA, Cavanagh HD, et al. Induction of alpha-smooth muscle actin expression and myofibroblast transformation in cultured corneal keratocytes. Cornea 1996;15:505-16.

27 Serini G, Gabbiani G. Mechanisms of myofibroblast activity and phenotypic modulation. Exp Cell Res 1999;250:273-83.

28 Serini G, Bochaton-Piallat ML, Ropraz P, et al. The fibronectin domain ED-A is crucial for myofibroblastic phenotype induction by transforming growth factor-betal. J Cell Biol 1998;142:873-81.

29 Jester JV, Petroll WM, Barry PA, et al. Temporal, 3-dimensional, cellular anatomy of corneal wound tissue. J Anat 1995; 186:301-11.

30 Jester JV, Petroll WM, Feng W, et al. Radial keratotomy. 1. The wound healing process and measurement of incisional gape in two animal models using in vivo confocal microscopy. Invest Ophthalmol Vis Sci 1992;33:3255-70.

31 Jester JV, Petroll WM, Cavanagh HD. Corneal stromal wound healing in refractive surgery: the role of myofibroblasts. Prog Retin Eye Res 1999:18:311-56.

32 Stratton R, Rajkumar V, Ponticos $M$, et al. Prostacyclin derivatives prevent the fibrotic response to TGF-beta2 by inhibiting the Ras/MEK/ERK pathway. FASEB J 2002;16:1949-51.

33 Folger PA, Zekaria D, Grotendorst G, et al. Transforming growth factor-betastimulated connective tissue growth factor expression during corneal myofibroblast differentiation. Invest Ophthalmol Vis Sci 2001;42:2534-41.

34 Frazier K, Williams S, Kothapalli D, et al. Stimulation of fibroblast cell growth, matrix production, and granulation tissue formation by connective tissue growth factor. J Invest Dermatol 1996:107:404-11.

35 Igarashi A, Nashiro K, Kikuchi K, et al. Connective tissue growth factor gene expression in tissue sections from localized scleroderma, keloid, and other fibrotic skin disorders. J Invest Dermatol 1996;106:729-33.

36 Møller-Pedersen T, Li HF, Petroll WM, et al. Confocal microscopic characterization of wound repair after photorefractive keratectomy. Invest Ophthalmol Vis Sci 1998;39:487-501.

37 Cintron C, Kublin CL. Regeneration of corneal tissue. Dev Biol 1977;61:346-57

38 Moller-Pedersen T. On the structural origin of refractive instability and cornea haze after excimer laser keratectomy for myopia. Acta Ophthalmol Scand 2003;81:1-20. 